\title{
Safety of Patient-Controlled
} Analgesia After Surgery in Children And Adolescents: Concerns And Potential Solutions

\author{
Don Daniel Ocay ${ }^{1,2}$, Annik Otis ${ }^{3,4}$, Alisson R. Teles ${ }^{2,5}$ and Catherine E. Ferland ${ }^{1,2,3,4,5,6 *}$ \\ ${ }^{1}$ Department of Experimental Surgery, McGill University, Montreal, QC, Canada, ${ }^{2}$ Shriners Hospitals for Children-Canada, \\ Montreal, QC, Canada, ${ }^{3}$ Department of Anesthesia, McGill University, Montreal, QC, Canada, ${ }^{4}$ Department of Anesthesia, \\ Montreal Children's Hospital, Montreal, QC, Canada, ${ }^{5}$ Integrated Program in Neuroscience, McGill University, Montreal, QC, \\ Canada, ${ }^{6}$ Child Health and Human Development, Research Institute-McGill University Health Centre, Montreal, QC, Canada
}

OPEN ACCESS

Edited by:

Francesco Morini,

Bambino Gesù Ospedale Pediatrico

(IRCCS), Italy

Reviewed by:

Elke Ruttenstock,

Hospital for Sick Children, Canada

Hiromu Miyake,

Shizuoka Children's Hospital, Japan

${ }^{*}$ Correspondence:

Catherine E. Ferland catherine.ferland@mcgill.ca

Specialty section:

This article was submitted to Pediatric Surgery,

a section of the journal

Frontiers in Pediatrics

Received: 26 July 2018 Accepted: 17 October 2018 Published: 06 November 2018

Citation:

Ocay DD, Otis A, Teles AR and

Ferland CE (2018) Safety of Patient-Controlled Analgesia After Surgery in Children And Adolescents: Concerns And Potential Solutions.

Front. Pediatr. 6:336.

doi: 10.3389/fped.2018.00336
Patient-controlled analgesia (PCA) is common practice for acute postoperative pain management. Postoperative PCA use decreases pain intensity and improves patient satisfaction when compared to non-PCA routes of medication administration. Although PCA has several advantages regarding efficacy and safety, adverse events remain a concern. Programming errors and protocols, patient monitoring, and PCA by proxy or with continuous infusion are recurring silent dangers of PCA use in children and adolescents. Innovative considerations need to be emphasized for future improvement of PCA devices for elective surgical patients. With technology within the healthcare setting advancing at a fast pace, smart pump technology is something to look forward to.

Keywords: patient-controlled analgesia, pediatrics, safety, surgery, concerns, solutions

\section{INTRODUCTION}

Patient-controlled analgesia (PCA) was clinically introduced to adults in 1971 to quickly and effectively relieve postsurgical pain. Today, PCA is common practice for managing acute pain of hospitalized children and adolescents in different contexts such as postoperative, oncology, trauma and palliative care (1). The PCA allows the patient to self-administer analgesics, usually intravenous opioids, by using a programmable computerized pump. Giving patients the control over analgesic drug administration allows for better titration to maximize pain relief and minimize risk of overdose (2). This active self-management of pain is an important factor concerning patients' psychological well-being since the patient can receive pain medication immediately without the need for a nurse to administer it. Use of PCA after surgery decreases pain intensity and improves patient satisfaction when compared to non-PCA routes of opioid administration (3).

Although PCA has several advantages in terms of efficacy and safety $(4,5)$, adverse events remain a concern (6). In a meta-analysis from the Cochrane database, it was demonstrated that the incidence of opioid-induced adverse events is similar in PCA compared to non-PCA, with the exception of pruritus, which was increased with PCA (3). Optimizing the safety of PCA use with children and adolescents without compromising its efficacy is still a challenge for researchers and developers (7). Selection and programming of the pump, monitoring and selection of patients, staff and patient/parent education, as well as the use of PCA by proxy are recurrent themes for safe PCA use. In this mini-review we will highlight safety issues of PCA use and suggest innovative considerations for future improvements of PCA pumps for elective surgical pediatric patients which is summarized in Table 1. 
TABLE 1 | Summary of safety concerns and potential solutions of patient-controlled analgesia.

\begin{tabular}{ll}
\hline Concerns & Solutions \\
\hline Programming errors & - Smart pumps with barcode scanning \\
- Son et al. (6) & - Computer provider entry \\
- Rishoej et al. (8) & - Establish reporting system \\
- Campoe et al. (9) & - Regular audits \\
Programming protocol & - Standardization of protocol \\
- Macintyre (10) & - Patient evaluation \\
- Craft (11) & - Risk stratification \\
& - Staff education \\
Patient monitoring & - Assessment of sedation level \\
- Niesters et al. (12) & - Smart pumps with oximetry and capnography \\
- Langhan et al. (13) & \\
- Ronen et al. (14) & \\
- Craft (11) & \\
- Freemantle et al. (15) & \\
- Jay et al. (16) & \\
PCA by proxy & - Radiofrequency thumb tags \\
- Howard et al. (17) & - Patient and family education \\
- Craft (11) & \\
- Anghelescu et al. (18) & \\
PCA with continuous infusion & - Establish pediatric pain services \\
- George et al. (19) & \\
- McNeely et al. (20) & \\
- Hayes et al. (21) & \\
\hline & \\
\hline
\end{tabular}

\section{THE SILENT DANGERS OF PCA USE IN CHILDREN AND ADOLESCENTS}

\section{Programming Errors}

PCA relies on the use of a program with different parameters such as bolus dose, lockout interval, hourly maximum dose and the drugs used, that will allow the patient to receive opioids in a predetermined pattern. Programming errors of the pump are therefore an important challenge concerning the safety of PCA (8). A recent retrospective study collecting the clinical records of 82,698 pediatric surgical patients demonstrated that $0.19 \%$ of cases experienced PCA device-related errors with the electronic programmable pump showing the highest error rate (6). The study also demonstrated that approximately $63 \%$ of cases with device-related errors experienced some type of adverse outcome such as nausea, vomiting, pruritus and inadequate analgesia. Although $0.19 \%$ may seem low, it represents 155 patients where errors could have been avoided and 96 patients whose preventable adverse events such as respiratory depression, may have been prevented (6). Most errors were associated with pump misprogramming which lead to serious unintended consequences such as oversedation, respiratory depression or undertreated pain. Since nurses oversee medication administration tasks and with the frequent distractions and interruptions occurring within the healthcare setting that increase the number of PCA medication errors, Campoe and Giuliano (9) investigated the impact of frequent interruption (2, 4 , or 6 interruptions) on nurses' PCA programming performance. A mean error rate of $4.12 \%$ (10 errors) was observed, and although it may seem lower than the 20\% of PCA medication errors reported in the United States of America (22), it reflects 10 patients who would have received incorrect medication dosing (9). Medication errors can pose a serious problem to the pediatric patient population because medication dosing is weight or surface area based (8). Although PCA devicerelated errors may not be frequent, they may prompt to serious consequences such as prolonged hospitalization and disabilities.

To address the issue of programming errors, dedicated smart pumps with barcode scanning of medications that sound and look alike may be useful during the initial set up and verification of the PCA program. Standard opioid concentration solutions should be used, and a verification system must be implemented to ensure that concentrations from the physician's order matches the nurse's programming and the pharmacy's stock. A medical information management system such as a computer provider order entry (CPOE) may enhance accuracy of medication orders and prevent programming errors (22). $\mathrm{CPOE}$ ensures that drug orders are accurate, performs drug allergy checks, and identifies drug interactions or wrong drug dosing (22). In an era of fast advances in technology and research on artificial intelligence, developing a system that could monitor a patient's demand or recognize their intention to treat their pain along with their vital signs and risks of adverse effects seems appropriate to look forward to. Future studies should evaluate the effectiveness of new technologies on PCA programming errors or adverse events in the clinical setting.

A reporting system needs to be established to know how many errors are made, what was the cause, and the frequency. This system would allow the pain services to reconsider their protocol and guidelines and may lead to a meeting with the staff to search for solutions to improve their quality of care. Regular audits should be implemented for statistical purposes to know the frequency of adverse events and medication errors related to PCA. This would allow the hospital to think critically for improvements in their establishment to minimize any future adverse events.

\section{Programming Protocol}

Another clinical challenge of PCA use is the lack of consensus on the optimal PCA parameter programming in the pediatric population (10). For example, the lockout interval of PCA is commonly set at $6 \mathrm{~min}$ based on the onset of action of most opioids being of $5 \mathrm{~min}$. However, it is known that the peak effect of opioids varies between 7 and $15 \mathrm{~min}$ (11). Therefore, a 6-min lockout interval allows the patient to receive another dose of medication before the peak effect is reached. As a consequence, the patient is at risk for oversedation and serious adverse events such as respiratory depression.

Theoretically, a lockout interval of $10 \mathrm{~min}$ would allow for the full analgesic effect of opioids to take place before the next bolus dose is administered, decreasing overmedication and associated adverse events. However, previous studies have demonstrated that the influence of the lockout interval on pain relief and adverse effects had little to no effect $(23,24)$. Therefore, more studies regarding the influence of the lockout interval or 
the mechanism of action of opioids in PCA in children and adolescents are needed.

Preoperative self-reported measures that assess global psychological and behavioral profiles of the patients will identify those at risk of PCA misuse and adverse effects. This will allow for personalized PCA programming for patients in situations where they feel they have lost control such as after a surgery. In a case reported by Khan et al., psychiatric evaluation of an adolescent patient who received inadequate pain management revealed that the motivation behind increasing PCA doses was also to reduce anxiety. This behavior may complicate transition from PCA to oral analgesics and may cause the development of dependence during the arrest of medication (25). Online accessible validated tools assessing psychosocial parameters in the pediatric population are available and free of charge. Anxiety levels and pain catastrophizing, defined as exaggerating the pain experience than the general population, are among the best psychological predictors of patient's pain management after surgery $(26,27)$. Moreover, a preoperative psychophysical evaluation of the patient's somatosensory functioning with the use of quantitative sensory testing may provide insight into the postoperative pain experience of patients and their PCA dose-demand ratio (28). As an example, it was reported that the efficacy of patients' descending inhibitory pain system tested with a conditioned pain modulation paradigm before surgery predicted $(P=0.001)$ morphine consumption after surgery (29), suggesting a clinical value of such evaluation before surgery. A patient who demonstrates a tendency of catastrophizing a pain experience and is highly anxious, and whose descending inhibitory pain control is considered suboptimal would benefit of more preoperative education, monitoring, reassurance and most probably of the co-administration of an alpha 2-adrenergic receptor agonist that enhances the activity of descending inhibitory pain pathways (30). A limitation to patient evaluation is the reliability of one evaluation tool for all pediatric age ranges. There are accessible tools validated for specific age ranges (31). Therefore, it must be ensured that the appropriate method for patient evaluation is used and assessed in identifying the risk of PCA use and adverse effects in further studies.

A quality improvement project Cincinnati Children's Hospital Medical Center (2017) used risk stratification to stratify more than $90 \%$ of their patients for opioid misuse allowing earlier intervention in patients considered high risk (32). Risk stratification using a combination of subjective and semiobjective evaluations before surgery would pinpoint the patients who will properly participate in the management of their pain and those who need close monitoring and additional education on safe PCA use.

\section{Patient Monitoring}

Opioid-induced respiratory depression is the most serious complication of PCA therapy. Although the incidence is low $(2.3 \%)$ (3), it may lead to respiratory arrest if not recognized and treated promptly $(12,33)$. Periodic spot checks of PCA patients remain the standard of care to detect opioid-induced respiratory depression and over-sedation. The issue with this method of monitoring is that patients are momentarily aroused by the patient-nurse interaction. Stimulation of the patient can transiently increase their respiratory rate and alertness and can result in an inaccurate reflection of their baseline clinical status. In this sense, an electronic safety net has been proposed for PCA patient monitoring with the use of both pulse oximetry and capnography. A study conducted by Langhan et al. analyzed the impact of capnography monitoring of 201 children in the post-anesthesia care unit. They observed that capnography monitoring leads to more effective staff interventions of respiratory depression (13). The Integrated Pulmonary Index (IPI) was recently developed and validated in a retrospective cohort of 523 patients in a variety of clinical settings (14). The IPI uses and integrates 4 parameters (end tidal $\mathrm{CO}_{2}$, respiratory rate, arterial oxygen saturation, and pulse rate) to improve respiratory monitoring. The IPI demonstrated good reliability in interpreting the respiratory status of patients in the validation cohort mainly consisting of adults and 1 pediatric cohort under procedural sedation (14). Unfortunately, the IPI has not been evaluated for use in the postoperative pediatric population. While capnography and the IPI are promising, these measures may not be the most effective to detect early opioid-induced respiratory depression, as hypoxia can occur in the absence of a low respiratory rate (11) and the overall result of a composite index may reflect certain individual components rather than the whole composite outcome (15). Therefore, other measures to improve patient monitoring must be considered including an increased surveillance of the patient and preoperative identification of risk factors for respiratory depression such as presence of respiratory problems, neurologic disease, renal disease, obesity, etc. $(12,16,33)$.

The goal is early detection of opioid induced respiratory depression. Assessment of sedation level is the most reliable way to prevent opioid-induced respiratory depression as oversedation usually precede respiratory depression (34). The Pasero Opioid Sedation Scale (POSS) has been validated in children $(11,35,36)$. It is a simple and economic tool with lifesaving benefits that can be easily incorporated into periodic spot checks of PCA patients. Surveillance with a safety checklist and daily documentation of PCA opioid trends should be implemented.

Better development of pediatric monitoring such as integrating the overall response to analgesia with reported pain intensity is essential to increase safe PCA use. An innovative proposition would be a smart pump with pulse oximetry and capnography that decreases and/or stops opioid administration when parameters go beyond a predetermined limit and alarms healthcare staff.

\section{PCA by Proxy}

A major human error can occur during PCA by proxy, defined as the activation of the PCA by someone other than the patient, most commonly family members, caregivers, or friends. This must be differentiated from nurse-controlled analgesia (NCA) which is also defined as PCA delivered by proxy. NCA is suitable when PCA is contraindicated, such as the patient is 
unable to understand the concept of PCA or does not wish to control their analgesia. Although NCA is not as safe as PCA, nurses are better trained in postoperative pain management in comparison to a family member, caregiver or friend (17). PCA by proxy other than a nurse can be a highly dangerous practice as it negates a key safety measure of PCA use which is that a sleeping or sedated patient will not press the PCA button for more opioid administration (11). The individual pressing the PCA button for the patient may be a well-intentioned family member or caregiver intending to prevent pain upon awakening. Although PCA by proxy has been reported to lead to high patient satisfaction and compliance of the proxy in cases of children under the age of 5, children with neuromuscular impairment, or during painful procedures $(18,37)$, it may also lead to oversedation of the patient and other adverse events if the patient receives more bolus doses than needed. PCA dose limits over a certain time period are programmed, but there is no reliable method to predict the proper amount of opioids a patient needs for pain relief or for life-threatening events (10).

With technology within the healthcare setting advancing at a fast pace, it is expected to soon observe changes in the PCA devices. One could think of the idea that, based on the concept of unlocking a cell phone with a fingerprint, radiofrequency identification thumb tags of the patient on the PCA button would decrease the risk of oversedation and adverse events caused by a secondary individual administering the medication instead of the patient.

Education is a key element to improve safety for PCA by proxy. Staff and patient/parent education need to be emphasized. For patient/parent education, written and verbal preoperative education should be emphasized, because postoperative education or reinstruction is only ideal if the patient is alert and in a calm environment. Parents must understand that the PCA pump should only be used by the patient. Parents can get involved in their child's pain management, but they need to understand that even though their child may self-report high pain levels, if the pain is tolerable, then additional PCA doses are not warranted. Keeping a calm environment may reduce their anxiety and in turn, reduce the anxiety of the patient $(38,39)$. Warning signs should be placed at the head of the bed or attached to the pump stating that PCA can only be used by the patient. Evaluating adequate comprehension after PCA education should be implemented to assess the understanding of the patient and their parents.

\section{REFERENCES}

1. Franson HE. Postoperative patient-controlled analgesia in the pediatric population: a literature review. AANA J. (2010) 78:374-8.

2. Verghese ST, Hannallah RS. Acute pain management in children. J Pain Res. (2010) 3:105-23. doi: 10.2147/JPR.S4554

3. McNicol ED, Ferguson MC, Hudcova J. Patient controlled opioid analgesia versus non-patient controlled opioid analgesia for postoperative pain. Cochrane Database Syst Rev. (2015). CD003348. doi: 10.1002/14651858.CD003348.pub3

4. Faerber J, Zhong W, Dai D, Baehr A, Maxwell LG, Kraemer FW, et al. Comparative safety of morphine delivered via intravenous route vs.

\section{PCA With Continuous Infusion}

PCA with background opioid infusion has been suggested as a solution for PCA by proxy, but studies on adults show that postoperative PCA with continuous infusion increased the risk of respiratory depression (19). Furthermore, limited data on continuous infusion in the pediatric population and low quality of studies has led to inconclusions for its advantage (19). A recent meta-analysis of randomized trials comparing PCA with and without background infusion showed that there was no significant difference in outcomes and background infusion did not pose an advantage over the use of PCA alone $(20,21)$. A simple but also important human error is mistaking the nurse bell for the PCA button. The patient will receive fewer bolus doses than allowed or needed, resulting in suboptimal pain control. Therefore, proper education of staff, caregivers and patients and signs at the bedside reinforcing the fact that the PCA button should only be activated by the patient is necessary.

Establishing pediatric pain services that are trained to recognize and assess, and safely and efficiently treat acute pain is ideal in every healthcare establishment. This pain service should be available $24 \mathrm{~h}$ per day, 7 days per week to ensure that any adverse events are treated in a timely response, and pain is managed thoroughly. The team should have a comprehensible treatment plan for each patient that is accessible to facilitate communication between the rest of the medical team (40).

\section{CONCLUSION}

In summary, although PCA use has many advantages, concerns toward the safety of PCA devices remains. Careful patient selection and assessment, comprehensive education for patients, families and health care providers, PCA programming protocols with standardized solutions, and the use of appropriate monitoring are necessary for safe PCA use in the clinical setting. In addition, the practice should be regularly audited, and errors reported. As new technologies emerge, the use of smart pump technology and computer provider order entry will certainly decrease medication and programming errors.

\section{AUTHOR CONTRIBUTIONS}

DO reviewed the literature and drafted the mini-review. AO and AT reviewed the literature, wrote parts of the review and contributed to the critical revisions. CF conceptualized the manuscript and contributed to the critical revisions and approved the final version.

Patient-controlled analgesia device for pediatric inpatients. J Pain Symptom Manag. (2017) 53:842-50. doi: 10.1016/j.jpainsymman.2016.12.328

5. Chiaretti A, Genovese O, Antonelli A, Tortorolo L, Ruggiero A, Focarelli B, et al. Patient-controlled analgesia with fentanil and midazolam in children with postoperative neurosurgical pain. Childs Nerv Syst. (2008) 24:119-24. doi: 10.1007/s00381-007-0429-z

6. Son HJ, Kim SH, Ryu JO, Kang MR, Kim MH, Suh JH, et al. Devicerelated error in patient-controlled analgesia: analysis of 82,698 patients in a tertiary hospital. Anesth Analg. (2018). doi: 10.1213/ANE.00000000000 03397. [Epub ahead of print].

7. Nelson KL, Yaster M, Kost-Byerly S, Monitto CL. A national survey of American Pediatric Anesthesiologists: patient-controlled analgesia and 
other intravenous opioid therapies in pediatric acute pain management. Anesth Analg (2010) 110:754-60. doi: 10.1213/ANE.0b013e3181c a749c

8. Rishoej RM, Almarsdóttir AB, Christesen HT, Hallas J, Kjeldsen LJ. Medication errors in pediatric inpatients: a study based on a national mandatory reporting system. Eur J Pediatr. (2017) 176:1697-705. doi: $10.1007 / \mathrm{s} 00431-017-3023-8$

9. Campoe KR, Giuliano KK. Impact of frequent interruption on nurses patient-controlled analgesia programming performance. Hum Factors (2017) 59:1204-13. doi: 10.1177/0018720817732605

10. Macintyre PE. Safety and efficacy of patient-controlled analgesia. Br J Anaesth. (2001) 87:36-46. doi: 10.1093/bja/87.1.36

11. Craft J. Patient-controlled analgesia: is it worth the painful prescribing process? Baylor Univ Med Center Proc. (2017) 23:434-8. doi: 10.1080/08998280.2010.11928666

12. Niesters M, Overdyk F, Smith T, Aarts L, Dahan A. Opioid-induced respiratory depression in paediatrics: a review of case reports. Br J Anaesth. (2013) 110:175-82. doi: 10.1093/bja/aes447

13. Langhan ML, Li F-Y, Lichtor JL, Lerman J. The impact of capnography monitoring among children and adolescents in the postanesthesia care unit: a randomized controlled trial. PAN Pediatric Anesth. (2017) 27:385-93. doi: 10.1111/pan.13077

14. Ronen M, Weissbrod R, Overdyk FJ, Ajizian S. Smart respiratory monitoring: clinical development and validation of the IPI (Integrated Pulmonary Index) algorithm. J Clin Monit Comput. (2017) 31:435-42. doi: 10.1007/s10877-016-9851-7

15. Freemantle N, Calvert M, Wood J, Eastaugh J, Griffin C. Composite outcomes in randomized trials: greater precision but with greater uncertainty? JAMA (2003) 289:2554-9. doi: 10.1001/jama.289.19.2554

16. Jay MA, Thomas BM, Nandi R, Howard RF. Higher risk of opioid-induced respiratory depression in children with neurodevelopmental disability: a retrospective cohort study of 12904 patients. Br J Anaesth. (2017) 118:239-46. doi: $10.1093 / \mathrm{bja} /$ aew 403

17. Howard RF, Lloyd-Thomas A, Thomas M, Williams DG, Saul R, Bruce E, et al. Nurse-controlled analgesia (NCA) following major surgery in 10,000 patients in a children's hospital. Paediatr Anaesth. (2010) 20:126-34. doi: 10.1111/j.1460-9592.2009.03242.x

18. Anghelescu DL, Kaddoum RN, Oakes LL, Windsor KB, Faughnan LG, Burgoyne LL. An update: the safety of patient-controlled analgesia by proxy for pain management in pediatric oncology: 2004 to 2010. Anesth Analg. (2011) 113:1525-6. doi: 10.1213/ANE.0b013e318234a388

19. George JA, Lin EE, Hanna MN, Murphy JD, Kumar K, Ko PS, et al. The effect of intravenous opioid patient-controlled analgesia with and without background infusion on respiratory depression: a meta-analysis. $J$ Opioid Manag. (2010) 6:47-54. doi: 10.5055/jom.2010.0004

20. McNeely JK, Trentadue NC. Comparison of patient-controlled analgesia with and without nighttime morphine infusion following lower extremity surgery in children. J Pain Symptom Manag. (1997) 13:268-73. doi: 10.1016/S0885-3924(96)00324-7

21. Hayes J, Dowling JJ, Peliowski A, Crawford MW, Johnston B. Patientcontrolled analgesia plus background opioid infusion for postoperative pain in children: a systematic review and meta-analysis of randomized trials. Anesth Analg. (2016) 123:991-1003. doi: 10.1213/ANE.00000000000 01244

22. Prewitt J, Schneider S, Horvath M, Hammond J, Jackson J, Ginsberg B. PCA safety data review after clinical decision support and smart pump technology implementation. J Patient Saf. (2013) 9:103-9. doi: 10.1097/PTS.0b013e318281b866

23. Badner NH, Doyle JA, Smith MH, Herrick IA. Effect of varying intravenous patient-controlled analgesia dose and lockout interval while maintaining a constant hourly maximum dose. J Clin Anesth. (1996) 8:382-5. doi: 10.1016/0952-8180(96)00077-3

24. Ginsberg B, Gil KM, Muir M, Sullivan F, Williams DA, Glass PS. The influence of lockout intervals and drug selection on patientcontrolled analgesia following gynecological surgery. Pain (1995) 62:95-100. doi: 10.1016/0304-3959(94)00255-D
25. Khan NA, Sullivan MA, Vitale MG, Tresgallo ME, Saroyan JM. Undetected Ultracet dependence in an adolescent with nonmalignant back pain. J Opioid Manag. (2013) 9:225-30. doi: 10.5055/jom.2013.0163

26. Logan DE, Rose JB. Is postoperative pain a self-fulfilling prophecy? Expectancy effects on postoperative pain and patient-controlled analgesia use among adolescent surgical patients. J Pediatr Psychol. (2005) 30:187-96. doi: 10.1093/jpepsy/jsi006

27. Esteve R, Marquina-Aponte V, Ramirez-Maestre C. Postoperative pain in children: association between anxiety sensitivity, pain catastrophizing, and female caregivers' responses to children's pain. J Pain (2014) 15:157-68.e1. doi: 10.1016/j.jpain.2013.10.007

28. Holbech JV, Bach FW, Finnerup NB, Jensen TS, Sindrup SH. Pain phenotype as a predictor for drug response in painful polyneuropathy-a retrospective analysis of data from controlled clinical trials. Pain (2016) 157:1305-13. doi: $10.1097 / j$.pain. 0000000000000563

29. Grosen K, Vase L, Pilegaard HK, Pfeiffer-Jensen M, Drewes AM. Conditioned pain modulation and situational pain catastrophizing as preoperative predictors of pain following chest wall surgery: a prospective observational cohort study. PLoS ONE (2014) 9:e90185. doi: 10.1371/journal.pone.0090185

30. Lambert P, Cyna AM, Knight N, Middleton P. Clonidine premedication for postoperative analgesia in children. Cochrane Database Syst Rev. (2014) CD009633. doi: 10.1002/14651858.CD009633.pub2

31. Ravens-Sieberer U, Karow A, Barthel D, Klasen F. How to assess quality of life in child and adolescent psychiatry. Dialogues Clin Neurosci. (2014) 16:147-58.

32. Thienprayoon R, Porter K, Tate M, Ashby M, Meyer M. Risk stratification for opioid misuse in children, adolescents, and young adults: a quality improvement project. Pediatrics (2017) 139:e20160258. doi: $10.1542 /$ peds.2016-0258

33. Gupta K, Prasad A, Nagappa M, Wong J, Abrahamyan L, Chung FF. Risk factors for opioid-induced respiratory depression and failure to rescue: a review. Curr Opin Anaesthesiol. (2018) 31:110-9. doi: $10.1097 /$ ACO.0000000000000541

34. Gregoire M, Paquette G, Bourgault P, Lapre J, Bell L, Lafrenaye S. Douleur aiguë pédiatrique : de l'urgence à l'unité de soins. Perspect Infirm. (2018) 15:61-7.

35. Pasero C, Manworren RC, McCaffery M. PAIN Control: IV opioid range orders for acute pain management. Am J Nurs. (2007) 107:52-9; quiz 9-60. doi: 10.1097/00000446-200702000-00023

36. Quinlan-Colwell A, Thear G, Miller-Baldwin E, Smith A. Use of the Pasero Opioid-induced Sedation Scale (POSS) in pediatric patients. J Pediatr Nurs. (2017) 33:83-7. doi: 10.1016/j.pedn.2017.01.006

37. Anghelescu DL, Zhang K, Faughnan LG, Pei D. The safety and effectiveness of patient-controlled analgesia in outpatient children and young adults with cancer: a retrospective study. J Pediatr Hematol Oncol. (2015) 37:378-82. doi: 10.1097/MPH.0000000000000354

38. Bearden DJ, Feinstein A, Cohen LL. The influence of parent preprocedural anxiety on child procedural pain: mediation by child procedural anxiety. $J$ Pediatr Psychol. (2012) 37:680-6. doi: 10.1093/jpepsy/jss041

39. Rosenberg RE, Clark RA, Chibbaro P, Hambrick HR, Bruzzese JM, Feudtner $\mathrm{C}$, et al. Factors predicting parent anxiety around infant and toddler postoperative and pain. Hosp Pediatr. (2017) 7:313-9. doi: 10.1542/hpeds.2016-0166

40. Kost-Byerly S, Chalkiadis G. Developing a pediatric pain service. Paediatr Anaesth. (2012) 22:1016-24. doi: 10.1111/pan.12004

Conflict of Interest Statement: The authors declare that the research was conducted in the absence of any commercial or financial relationships that could be construed as a potential conflict of interest.

Copyright (๑) 2018 Ocay, Otis, Teles and Ferland. This is an open-access article distributed under the terms of the Creative Commons Attribution License (CC BY). The use, distribution or reproduction in other forums is permitted, provided the original author(s) and the copyright owner(s) are credited and that the original publication in this journal is cited, in accordance with accepted academic practice. No use, distribution or reproduction is permitted which does not comply with these terms. 